\title{
V20. EVALUATION OF SCHEDULED MRM PARAMETERS. A POWERFUL TOOL FOR ANALYSIS OF MODIFIED CARRIER PROTEIN OF MENINGOCOCCAL POLYSACCHARIDE C CONJUGATE VACCINE.
}

Marilza Batista Corrêa ${ }^{1}$; Renata Chagas Bastos ${ }^{1}$; José Godinho da Silva Junior ${ }^{2}$; Elza C. S. Fiigueira ${ }^{1}$;Maria de Lourdes M. Leal ${ }^{1}$; Ivna Alana F.B. da Silveira ${ }^{1}$.

${ }^{1}$ Laboratório de Tecnologia Bacteriana, Bio-Manguinhos / Fiocruz;

${ }^{2}$ Laboratório de Macromoléculas, Bio-Manguinhos / Fiocruz.

INTRODUCTION Liquid Chromatography, Multiple Reaction Monitoring by Mass Spectrometry (LC-MRM-MS) method, using a stable isotope-labeled internal standard has shown major progress in biological analysis. In this approach, optimizing the selected precursor ion in Q1 and product ion in Q3, for each monitoring transition, is important for establishing the most sensitive MRM assay. The sensitivity and accuracy improvement requires an experimental study for determination of optimal instrumental parameters, in order to generate abundant quantities of precursor and product ion pair. The use of LC allows a fast separation, generating narrow peaks, reproducible retention time and peak area. The control of chromatographic parameter is performed by system suitability test. The use of Scheduled MRM requires determination of dwell and MRM detection window. The dwell time is the time spent for recording the signal intensity of one data point; a longer dwell time results in more accurate measurements of signal intensity. All these instrument settings are important to determine the exact amount of scans time of a particular event, leading to the best quality and quantity data possible.

OBJECTIVE Comparativestudy of accuracy observed inautomatic Scheduled MRM and manually set up:evaluation by amino acid (Glu/Asp) standard solution analysis.

METHODOLOGY The MRM detection window, dwell and retention windowtimeswere optimized for LC-MS system (API 3200; Sciex). The chromatographic separation was carried out withan Agilent Column (C18; $4,6 \times 150 \mathrm{~mm}, 5 \mu \mathrm{m})$, in gradient mode. System suitability was performed using a commercial amino acid solutionAtraq ${ }^{\mathrm{TM}}$ (Sciex).A solution of isotope-labeled aspartic and glutamic acids, in concentration range from 0.25 to $5 \mathrm{pmol} / \mu \mathrm{L}$, was used as control. 
RESULTS Acquired data for treated aminoacids (Glu/Asp) were provided by Analyst Software Scheduled MRM ${ }^{\mathrm{TM}}$ Algorithm(method 1) and two others manual MRM methods: dwell time calculated for each analyte (method 2) and MRM detection window using $120 \mathrm{~ms}$ (method 3). Data were analyzed using statistic program GRAPHP ad PRISMA. For comparison among methods, a regression analysis was performedby least squares method. The standard deviations results of the linear adjust (Sy.x) calculated for three methods were in area units (AU): method $1(29,350)$ and methods 2, 3 (9000) for Asp; method 1 (14,030) and methods 2,3 (5000) for Glu; this represents a decrease about $60 \%$. The better correlation coefficient $\left(\mathrm{r}^{2}\right)$ was for the method 3 (0.99) showing acceptable statistical relationship between the concentration and observed data for both amino acids; others methods showed $\mathrm{r}^{2}$ values out of acceptable range.

CONCLUSION Statically, the use of MRM detection window of $120 \mathrm{~ms}$ (method 3) resulted in more accuratedata and will be further used inthe compilation of data generated by amino acid analysis ofcarrier protein present in meningococcal $\mathrm{C}$ conjugate vaccine.

KEYWORDS MRM/LC/MS, amino acid analysis. 